\title{
To prompt or not to prompt: A descriptive study of journaling practices used by nursing students during study abroad trips
}

\author{
Paulette A. Chaponniere, Laura E. Hall \\ Kirkhof College of Nursing, Grand Valley State University, Grand Rapids, Michigan, USA
}

Received: October 1, 2018

DOI: $10.5430 /$ jnep.v9n8p46
Accepted: April 9, 2019

Online Published: April 26, 2019

URL: https://doi.org/10.5430/jnep.v9n8p46

\begin{abstract}
Background: Reflective journaling has been widely used as an education tool. Minimal research has occurred to determine whether prompted or open format journaling nurtures cultural competence.

Methods: The efficacy of journal prompts as a pedagogical tool was assessed in this descriptive study. Undergraduate nursing students $(\mathrm{N}=49)$ reflected on their clinical experiences during 2-week trips to Ghana over 4 years $(2013-2016)$. The 1st, 2nd and 4th trip, students were assigned open reflective journaling. The 3rd trip, students were given prompted questions to answer. Entries were coded using Atlas.ti 7.

Results: The prompted format produced shorter entries with less rich and reflective substance. Some entries only allowed for two codes. Unprompted entries provided up to 28 codes.

Conclusions: During cross-cultural encounters, unprompted journaling may produce the richest results. When prompts are used, the format needs to be carefully selected to facilitate transformative learning.
\end{abstract}

Key Words: Cross-cultural, Reflective journaling, Nursing, Transformative learning

\section{BACKGROUND}

Reflective journaling is commonly used as an educational tool for students experiencing high-impact learning. Journal entries are also a useful data collection method. ${ }^{[1-5]}$ Journaling is frequently used in nursing education. This tool is effective to inspire students' introspective thinking process which can improve self-reflection, critical thinking, and cultural awareness during clinical experiences. ${ }^{[6-8]}$ Improving these skills can contribute to better self-awareness and a broader worldview in order to produce culturally sensitive, resilient nurses.

Different journaling formats have been suggested in pre- vious literature. These include open reflective journaling ${ }^{[9]}$ prompted journaling ${ }^{[6]}$ and freestyle journaling. ${ }^{[7,10]}$ Though there are some recommendations in literature, there is a lack of conclusive research defining the most effective style of journaling for data collection or educational reflection. No studies were found that determined which types of specific prompts would produce rich reflections.

The undergraduate Bachelor of Science in Nursing (BSN) program in this institution was modified to a concept-based curriculum one year prior to the first study abroad trip. The aim of this change was to streamline theory content by using exemplars to illustrate key components of nursing care. Some

*Correspondence: Paulette A. Chaponniere; Email: chaponnp@gvsu.edu; Address: Kirkhof College of Nursing, Grand Valley State University 301 Michigan St NE, Grand Rapids, USA. 
concepts, such as culture, were presented in the introductory nursing courses and incorporated in subsequent courses. Journaling was also introduced as a pedagogical teaching method in the first nursing course. Faculty used a variety of prompts similar to those developed by Tanner ${ }^{[11]}$ to guide students in self-reflection (see Table 1). Subsequent courses continued to use this reflective practice through prompts. Application varied by clinical course.

The college of nursing in this university placed high value on cross-cultural experiences. Administration and faculty supported the development of a clinical experience in Ghana, Africa, which would fulfill community health nursing course requirements. Eligible students were in either of their last two semesters of the undergraduate nursing program. The focus of the clinical experience was on global health and the implementation of a health education session in elementary schools. Students were required to write journal entries. These journal entries provided data for a multi-faceted study that analyzed nursing students' journal entries written during a 2-week experience in Ghana. Four separate 2-week programs from 2013 to 2016 were assessed and compared. The quality and depth of journal entries will be discussed in this article.

\section{Literature REVIEW}

\subsection{Travel journaling: A time-honored practice}

Travel journals have been kept by world explorers since voyages began. Many documented their observations and experiences when interacting with cultures other than their own. These narratives have been used to help students understand motivations in tourism, ${ }^{[12]}$ the impact of travel accounts on major European movements such as the Enlightenment ${ }^{[13]}$ and culture in general. ${ }^{[14]}$ Heuer, Russell and Kahlstorf ${ }^{[15]}$ identified that journals were a useful instrument for data collection in transcultural nursing research. Ethnonursing research methods developed by Leininger and McFarland ${ }^{[16]}$ incorporate the use of journals as a qualitative data collection tool. Journaling is thus a teaching and research method with a history of use as nursing students encounter other cultures. ${ }^{[17]}$

\subsection{Reflective practice: A key to growing professional expertise}

Many authors have focused on the benefits of journaling which include improvement of entries over time, growth of nursing characteristics, cultural awareness, and personal understanding. ${ }^{[1-3]}$ Lauterbach and Hentz ${ }^{[5]}$ summarized the benefits of journaling as a form of reflection such as revisioning reflection, reflection on experience as a universal Published by Sciedu Press human process, and reflection in action. When reflective journaling is paired with clinical experience, it can improve students' critical thinking, self-reflection, and cultural humility. ${ }^{[7,18]}$ Other benefits of journaling include discovering meaning, making connections between experiences and the classroom, clarifying values, gaining others' perspectives, reflecting on professional roles, improving writing skills, developing critical thinking, developing affective skills, and caring for self. ${ }^{[1,8]}$

Frequent and supportive faculty feedback on journaling was found to be important for student growth. ${ }^{[3,4,8]}$ Journaling is also a way to personalize education. Journal entries give faculty an opportunity to review student thinking, uncover misunderstandings, trace student thinking in the context of care, and assess student development of rationales for care. ${ }^{[4,5]}$

\subsection{Use of prompts}

Educators have had a wide range of approaches to the specifics of journaling from open-ended journaling to specific prompted entries. Epp ${ }^{[19]}$ noted in a systematic review that though journaling has been accepted as a useful form of introspective education, the most valuable and productive form has not yet been determined. Some researchers found that specific templates and questions provided appropriate journal entries. ${ }^{[4,20]}$ Others recommended that students adopt either a free writing approach or open-ended questions. ${ }^{[7,10]}$

Literature indicates that faculty guidance can help students develop reflective skills. ${ }^{[1,4,8,9]}$ Several models exist to assist students in their reflective journaling. Some provide specific prompts while others suggest a mixed approach. Tanner, ${ }^{[11]}$ Fakude and Bruce, ${ }^{[9]}$ and Lasater and Nielsen ${ }^{[4]}$ used specific prompts. Lasater and Nielsen ${ }^{[4]}$ proposed a clinical judgment model. They compared it to Tanner's ${ }^{[11]}$ model and recommended prompts to guide faculty's analysis of student journal entries to determine growth in clinical experiences. Williams ${ }^{[20]}$ provided specific questions for students to use during their psychiatric rotation and found that the reflective journaling process improved students' self-awareness of personal biases and preconceived notions. Some educators advocated for a mix of both approaches. O'Reilly and Milner ${ }^{[7]}$ proposed a mixed method to include e-journals, group blogs, and on-line reflective summaries. Sewell ${ }^{[21]}$ discussed thinking-in-action (i.e., journaling with questions), reflective writing (i.e., reflective dialogue), and freestyle journaling (i.e., less introspective, more cathartic) as types of journaling to improve thinking, confidence, and transition for student nurses. There does not appear to be a consensus in the literature on the most effective format for journaling. 


\subsection{Challenges for effective journaling}

Journaling is a useful source for qualitative data. ${ }^{[1,6,22]}$ Faculty can utilize this information for trip planning and to identify the flaws in implementing learning experiences. Faculty members are then able to carry out appropriate strategies to improve education programs. Hayman, Wilkes, and Jackson $^{[22]}$ identified challenges when researchers used journaling as a data collection method. These included poor participation, students feeling exposed, and not staying on track. Van Horn and Freed ${ }^{[23]}$ found that students placed in pairs for journaling had higher reflection rates and lower anxiety themes in their journaling than students who wrote individually. Journaling challenges also include student acceptance and participation. Hendrix, O'Malley, Sullivan, and Carmon ${ }^{[24]}$ noted that students valued the following attributes in journaling (in descending order of importance): time, anonymity, semi-structured or open free-form and receiving feedback at least one time from faculty members. Ruiz-Lopez et al. ${ }^{[10]}$ recommended that students adopt a free writing approach or be guided by open-ended questions.

\subsection{Faculty role}

Applying journaling in a useful and valued format is the role of the faculty. Effective journaling requires appropriate input from faculty as well as time commitment. ${ }^{[4,6,9,10,24]}$ In order to monitor the quality of entries, faculty should offer feedback over short time periods, and the feedback needs to be constructive in nature. Feedback fosters a dialogue between faculty and students. Blake ${ }^{[1]}$ recommended that faculty schedule an informative meeting with students to review written guidelines for journaling. He also advised a personal interview with each student at the start of the activity. This interaction provides faculty an opportunity to discover the students as individuals. Blake ${ }^{[1]}$ also deemed that "[f]aculty owe students a careful reading of entries" ( $p$. 9).

\subsection{Theoretical framework: Transformative learning theory}

The transformative learning theory was used as the framework for this study. Mezirow ${ }^{[25]}$ defines transformative learning as "learning that transforms problematic frames of reference... to make [learners] more inclusive, discriminating, open, reflective, and emotionally able to change" (p. 58). The 10 steps of Mezirow's early transformative learning theory include identifying a disorienting dilemma; self-examination with feelings of shame or guilt; critical assessment of epistemic, sociocultural, or psychic assumptions; relating personal discontent and the process of transformation to others; exploration of options for new roles, relationships and behavior; planning a course of action; acquisition of knowl- edge and skills to implement plans; experimenting with new roles; building confidence in new roles and relationships; and reintegration into one's life based on a new self-concept. ${ }^{[26]}$ Journaling is used by faculty as a tool of critical reflection to help foster transformative learning in nursing students. This transformative learning can initiate student self-examination, help them build confidence, and alter their worldviews. This process is a key component to growing cultural competence.

\section{METHOD}

This descriptive study assessed the effectiveness of journaling as a pedagogical tool. Institutional Review Board (IRB) approval was obtained from the university. Nursing students attending 2-week trips to Ghana were given reflective journaling assignments for pedagogical insight and data collection. Students were notified that their entries would be assessed by faculty to improve study abroad trips.

\subsection{Sample}

Undergraduate nursing students in a traditional bachelor of science in nursing (BSN) 4 years program, second degree (SD) program or in a registered nurse $(\mathrm{RN})$ to $\mathrm{BSN}$ program were offered the opportunity to study abroad. Those interested also needed to meet the study abroad requirements of the university. Applications were reviewed by the study abroad team. After acceptance, students were required to attend two orientation sessions state-side and another one on site in Ghana. Expectations of the immersion experience were reviewed prior to departure and clarified as needed while in Ghana.

\subsection{Data collection}

Data was collected from four separate travel groups from 2013-2016. A few students on each trip preferred to manually write their reflections, while most submitted them electronically. Hand-written journal entries were transcribed to an electronic format before being coded. Rigor was maintained. All entries were de-identified and data stored safely. A log of decisions made during the coding was kept.

Journal prompts varied by trip. For the first two trips, students were instructed to use an open format for their journal entries (see Table 1). On the third trip, in an effort to be consistent with all nursing clinical groups (i.e., medicalsurgical, mental health, and community health), specific journal prompts were initiated (see Table 2). Due to the short entries provided on the third trip, faculty decided to use the open format for the fourth trip which focused on emotional responses (see Table 3). For trips two, three and four, students were asked to write a final journal with specific reflection prompts approximately 1 month after returning to the United States (see Table 4). 
Table 1. Journal prompts for first community health nursing program

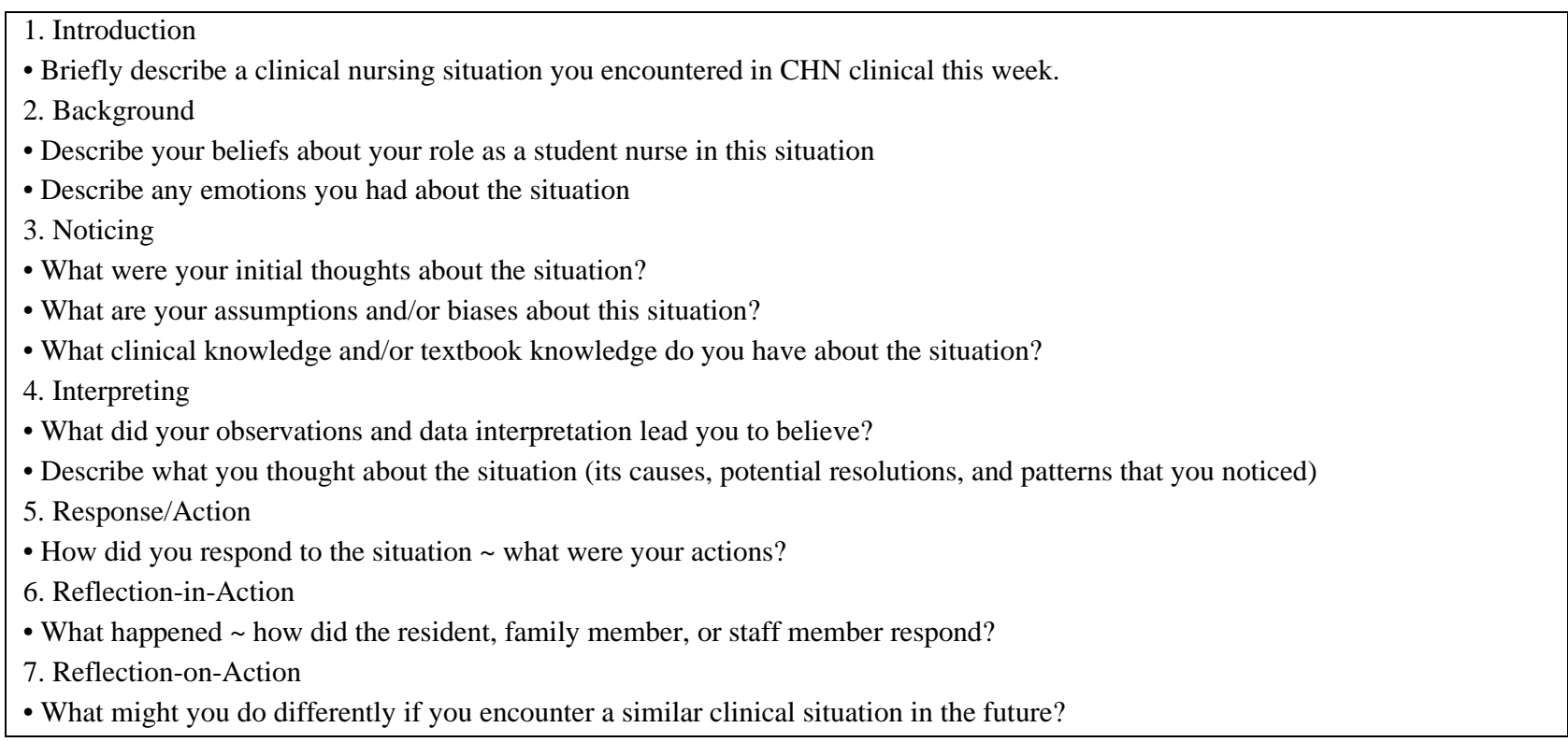

Table 2. Guides for Journaling in Trips 1 (2013), 2 (2014), and 4 (2015)

Describe your day in your diary. Then, based on one of the
situations you experienced, add a journal entry using one of the
following statements.
1. I was surprised that ...
2. I felt sad that...
3. I was delighted when....
4. I felt angry that...
5. I felt uncomfortable when...
6. I felt guilty that....
7. I was shocked when....
8. I was glad that....
9. I was pleased when.....
10. I enjoyed......because....

Table 3. Journal prompts for Trip 3 (2015)

1. What was the best part of your week?
2. If you could do your clinical time over again this week, what
would you do over?
3. How did you feel about clinical this week?
4. How did you include your aggregate or clients in decisions
about care this week?
5. What are your goals for next week?
6. Is there anything that I can do differently to help facilitate your
learning?

In order to promote sustainability of the program, faculty rotated. A faculty member who had previously travelled to Ghana went with a faculty member who had not (see Table 5). This created a larger pool of prepared trip directors.

\subsection{Data analysis}

To ensure accuracy, the researchers followed the data analysis process described by De Chesay. ${ }^{[27]}$ After each trip, two researchers separately coded entries using Atlas.ti 7. The first level of coding required that the researchers read the journal entries several times before labeling segments of the journals. After coding each set of entries, the researchers reviewed selected codes to determine if there were redundancies and modified codes when deemed necessary. The second level of coding required that composites of group data be created. Researchers grouped comments by common themes and meanings into types of learning: affective, behavioral and cognitive. A total of 133 possible codes were assigned to the 512 journal entries. The hierarchy and subcategories of coding used are noted in Figure 1.

Table 4. Final journal entry prompts for Trips 2 (2014), 3 (2015), and 4 (2016)
1. What was the most memorable part of the experience?
2. What annoyed you most?
3. What emotional challenges did you face?
4. What physical challenges did you face?
5. What moved your spirit?
6. How have you been changed by this experience?
7. How was your re-entry into the U.S.?

\section{Results}

\subsection{Demographics}

The majority of students who participated were in a traditional BSN program (71\%) followed by second degree students (24\%) and RN to BSN program (5\%). Only three males participated in these study abroad trips. The number of 
students travelling each trip as well as the number of journal entries per trip fluctuated (see Table 5). Other variations were age (from 22 to 50 years), experience (in international travel and nursing), and education background.
The ratio of faculty to student differed by trip (see Table 5 ). Even though this institution requires a ratio of 1 faculty member to 12 students for study abroad trips, the nursing education directive of 1:8 was accepted.

Table 5. Journal entry statistics

\begin{tabular}{lllllllllll}
\hline Year & Trip & Students & $\begin{array}{l}\text { Journal } \\
\text { Entries } \\
\text { per } \\
\text { Student }\end{array}$ & $\begin{array}{l}\text { Total } \\
\text { Number } \\
\text { of Entries }\end{array}$ & $\begin{array}{l}\text { Total } \\
\text { Number } \\
\text { of Codes }\end{array}$ & $\begin{array}{l}\text { Average } \\
\text { Codes } \\
\text { per Entry }\end{array}$ & $\begin{array}{l}\text { Post-trip } \\
\text { Entry }\end{array}$ & $\begin{array}{l}\text { Average } \\
\text { Codes per } \\
\text { Post-trip } \\
\text { Entry }\end{array}$ & $\begin{array}{l}\text { Scripted } \\
\text { Prompts }\end{array}$ & $\begin{array}{l}\text { Faculty } \\
\text { Student } \\
\text { Ratio }\end{array}$ \\
\hline 2013 & 1 & 8 & 6 & 44 & 549 & 12.5 & $\mathrm{~N}$ & $\mathrm{~N} / \mathrm{A}$ & $\mathrm{N}$ & $1: 4$ \\
2014 & 2 & 13 & 5 & 64 & 946 & 14.8 & $\mathrm{Y}$ & 17.3 & $\mathrm{~N}$ & $1: 6$ \\
2015 & 3 & 12 & 13 & 153 & 1694 & 11 & $\mathrm{Y}$ & 23.1 & $\mathrm{Y}$ & $1: 6$ \\
2016 & 4 & 16 & 16 & 251 & 2877 & 11.5 & $\mathrm{Y}$ & 5.6 & $\mathrm{~N}$ & $1: 5$ \\
\hline
\end{tabular}

Note. 2013: 2 faculty (A and B); 2014: 2 faculty (C and D) Faculty A was onsite but with no clinical role; 2015: 2 faculty (D and E); 2016: 3 faculty (A, E and F). Faculty C had been on the exploratory trip, and Faculty B, retired before the second trip.

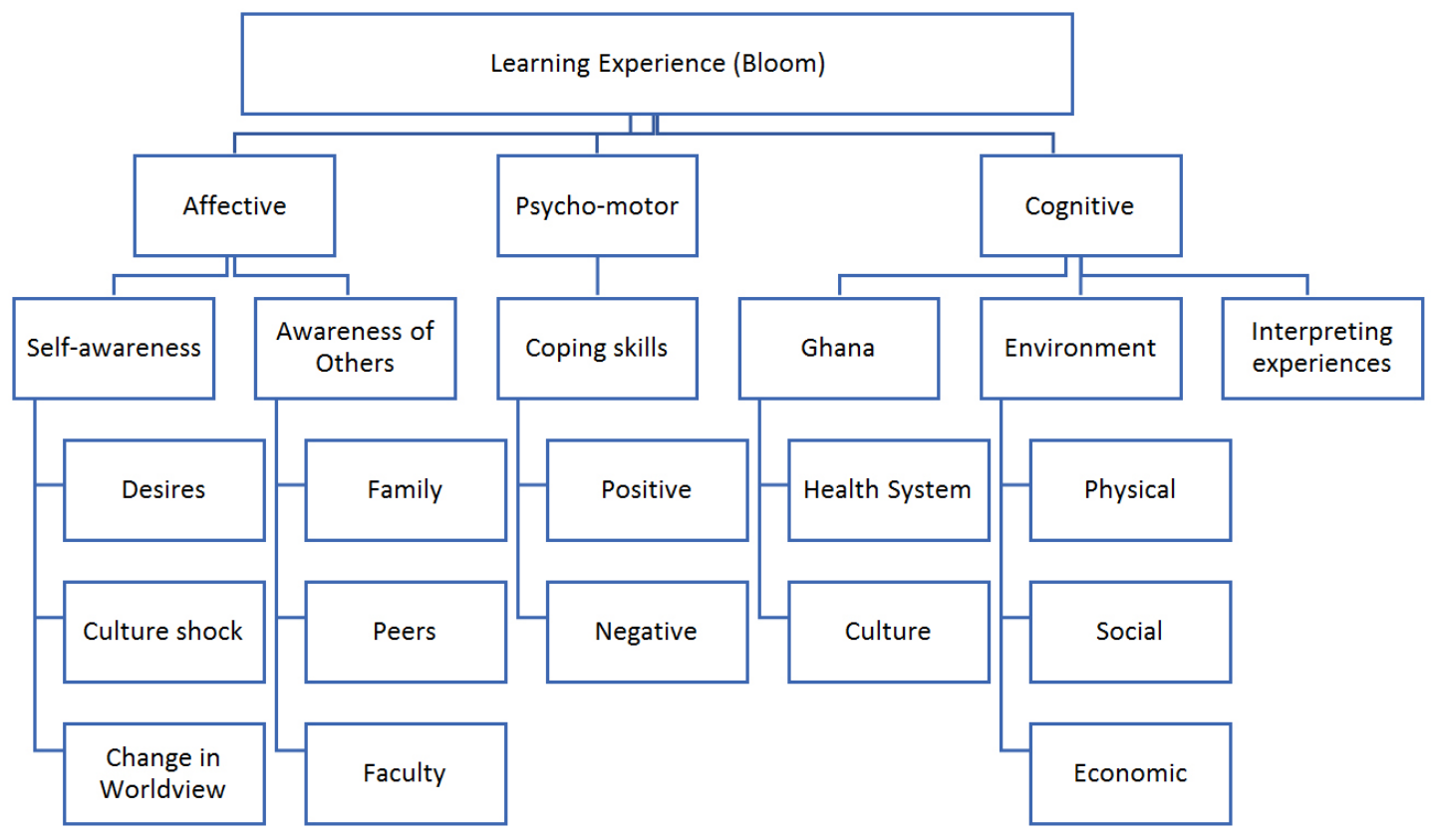

Figure 1. Coding hierarchy and subcategories using Atlas.ti 7

\subsection{Richness of data}

An unanticipated result was noticed while performing journal coding and comparing journal entries. Students who were provided with specific prompts on the third trip wrote shorter entries with less rich and reflective substance compared to those on the first, second and fourth trips with open-ended journaling formats. Some prompted entries written during the third trip only provided enough data for two codes versus some with data for 28 codes on other trips. Many entries on the third trip were one word or one sentence long.

Length of entries was congruent with depth of codes. Average word counts were compared between trips (see Table 6).
The third trip had dramatically shorter journal entries when compared to the first and second trip journals. However, the third trip had longer answers for final entries when compared to main entries and final entries from other trips. Though prompts did not assist with length and depth of answers for the main entries of the third group, it was beneficial for the final entry. The other two groups also had length and depth in their final entries when these prescriptive prompts were provided. Entries became slightly richer during the fourth trip with up to 32 codes per entry. Average coding of the post-trip entries was not congruent with trip entries. Number of codes per entry ranged from an average of 17.3 on trip two to 23.1 on trip three, and 5.6 on trip four (see Table 5). 
Table 6. Word count for journal entries

\begin{tabular}{lllll}
\hline Year & Total words & Number of entries & Average words per entry (excluding final entry) & Average words per final entry \\
\hline 2013 & 16,023 & 35 & 457 & NA \\
2014 & 41,190 & 64 & 643 & 796 \\
2015 & 40,194 & 142 & 283 & 1,015 \\
2016 & 80,856 & 236 & 343 & 509 \\
\hline
\end{tabular}

\section{DisCUSSION}

The quality and length of journal entries varied between trips. This could be attributed to several factors including student seniority, previous experience with journaling as a self-reflective strategy, faculty feedback, length of learning experience, and frequency of entries.

\subsection{Student seniority}

Students who were eligible to travel to Ghana had completed either three or four semesters of their undergraduate course work. For all previous clinical rotations, students had been required to journal about their experiences. This pedagog ical practice was also a requirement for study abroad trips. Students in the third cohort trip might have provided journal entries with richer reflection if they had not been given specific prompts. This finding would be supported by Hendrix et al. ${ }^{[24]}$ who noted that students in higher level courses preferred open format journaling in contrast to students in earlier courses who preferred prompts. O'Reilly and Milner ${ }^{[7]}$ found that fourth year students preferred more autonomous types of reflection.

\subsection{Previous experience in journaling}

Students' previous experience with journaling could also impact the quality of their entries. Some students informally shared that they loved to write. Others perceived journaling as "busy work". Using prompts rather than an unprompted option may have caused students to perceive the learning activity as an assignment to complete rather than as a tool to foster self-reflection. Sewell ${ }^{[21]}$ noted that freestyle journaling encourages introspection. Freestyle journaling which fosters transformative learning may have occurred but was not being demonstrated in the prompted journal entries during the third trip.

\subsection{Faculty feedback}

Fakude and Bruce ${ }^{[9]}$ recommended that journaling be supplemented by other educational strategies such as discussion groups to improve clinical judgment. Faculty feedback, whether written or verbal, motivates students to examine their clinical experiences. Feedback also provides data for faculty to assess implementation issues such as housing or personal health.

Published by Sciedu Press
Directors of the four study abroad trips to Ghana provided either verbal or written feedback on journal entries. Faculty also used face-to-face time for on-going dialogue with students. This often occurred while groups were being transported to and from clinical sites. Travel time provided an opportunity for more interaction between peers and between students and faculty to debrief their experiences. Students utilized this debriefing time to examine their experiences and receive faculty feedback before writing journal entries.

Frequency of faculty feedback may have also influenced the quality of journal entries. Some faculty read and provided feedback on each journal entry before the next one was written. Others read them at the end of the trip and then provided feedback. However, Hendrix et al. ${ }^{[24]}$ noted that students in their study were "indifferent to a single feedback or multiple feedbacks" (p. 5). Therefore this may not have been a determining factor in journal quality.

\subsection{Frequency of entries and length of experience}

The literature reviewed did not address the optimum number of journal entries needed to foster transformative learning. Previous researchers' requirements for journaling varied. Lasater and Nielsen ${ }^{[4]}$ used four guided, open reflections during a 10-week course: two at midterm and two at the end of the course. Ruis-Lopez et al. ${ }^{[10]}$ asked students to journal every 2 weeks. Tanner ${ }^{[11]}$ asked students to journal after simulation activities. Fakude and Bruce ${ }^{[9]}$ instructed students to select one clinical experience per week over 8 weeks. Sewell ${ }^{[21]}$ recommended reflection to facilitate transition into a new role as staff nurse after critical incidents but did not indicate the frequency. Dunlap ${ }^{[6]}$ found that weekly journal entries led to burnout. She proposed a bi-monthly schedule.

In this study, students wrote five to six entries for the first and second trips. For the third and fourth trips, students provided 13-16 entries. The rate of journaling was much higher in this study than in the studies reviewed in the literature. In comparing number of entries, the trips with a higher number of entries had a lower number of codes per entry. Requiring journal entries every day on the third and fourth trips could account for shorter and less rich data. Of note, the students who had followed specific prompts during the third 
trip provided the richest data for their prompted 1month post trip journal as compared to the other groups (see Table 5). This may indicate that it was the style of prompts used for the daily entries during the third trip rather than the use of prompts in general.

Most previous studies which used journal entries for selfreflection were implemented over the span of an entire semester or multiple semesters. Having time for reflection is an important factor in promoting transformative learning. ${ }^{[6,7,19]}$ Due to the brief nature of these trips, students had less free time to journal and less adaptive time to become comfortable in the Ghanaian culture. This could then impact the quality and length of their journal entries.

\subsection{Limitations}

Some limitations were identified in this study. Possible influences on the journaling experience include the rotation of faculty members as trip directors, the variation in number of students and faculty to student ratios, the relatively short 2 weeks time frame for the experience, and differences in student learning styles. Faculty variations included inconsistent journaling instructions, quality and frequency of feedback, training and experience in using journaling as a pedagogical tool. Some faculty had formal instruction in the use of journaling. Others had developed their own approach to journaling. However, the transformative learning process is a self-assessing and growing process. The need for frequent faculty feedback should not be required to enrich journal writing. Many authors ${ }^{[3,19,24]}$ indicate that faculty members do have an active role in guiding students in self-reflection. Providing feedback does take time and is to be viewed as a dialogue between faculty and student. ${ }^{[4,10,24]}$

The number of students travelling each trip varied as did the previous international travel experience of each student. The majority of students had not travelled abroad. The number of students could also affect group dynamics. From informal faculty feedback, smaller groups were noted to be more fully involved and interactive with each other while larger ones were split and worked based on class enrollment. A larger group could change the debriefing dynamics as well as overall experiences. This could affect the face-to-face discussions and interactions among students and with faculty. Another factor that could change trip dynamics is the variation in faculty to student ratios between trips (1:4 vs. 1:6). This would influence interactions between faculty and students.

A 2 week time frame may impact growth in self-reflection as learning and change takes time. The final journal entry for the last three trips was written approximately 1 month after returning to the US. The quality of these entries, even though more guided, was richer than all others. The time 52 to process an intense experience needs to be acknowledged. In many of the previous studies found in the literature on journaling outcomes, researchers used longer time frames (from 8 weeks to 1 semester) and fewer overall entries. ${ }^{[6,9,19]}$

Learning styles also varied between students. This can cause differences in processing experiences. Auditory learners may benefit more from verbal reflections compared to visual learners who prefer to write out their reflections. Some students preferred to hand-write their entries while others submitted them electronically. No differences in number of codes between hand-written versus electronic journals were noted. Entries were utilized differently by each student. Some students developed self-awareness as they interacted with a different culture. Others perceived the journal entries as a way to communicate to faculty how to improve the learning experience.

\section{Conclusion}

Nursing students completed community health hours during a 2-week study abroad program in Ghana. As part of their transformative learning process, students wrote reflective journal entries. These journals were coded using Atlas.ti 7 software. A comparison of number of codes and average word counts revealed a variation in length and depth of reflections. In this study, journaling using specific prompts similar to what Tanner ${ }^{[11]}$ proposed produced shorter, less contemplative and descriptive entries. Students who followed an unprompted, open journaling format provided richer selfreflections. During high-intensity cross-cultural encounters, unprompted journaling may produce the richest results. When prompts are used, the format needs to be carefully selected to facilitate transformative learning. The frequency and quality of faculty feedback as well as number of journal entries need to be ascertained. This information will assist nurse educators to create and utilize appropriate introspective tools for cross-cultural educational journeys. Faculty also needs to be trained in how to use journaling effectively as a pedagogical tool. Further research is indicated to define the most effective pedagogical journaling format for cross-cultural experiences.

\section{ACKNOWLEDGEMENTS}

The authors wish to thank the Kirkhof College of Nursing administration, faculty and staff for their support (including financial) of this research. They acknowledge that study abroad cannot be successful unless there is institutional commitment to encouraging students to become global citizens.

\section{CONFLiCtS OF INTEREST Disclosure}

The authors declare that there is no conflict of interest. 


\section{REFERENCES}

[1] Blake TK. Journaling: an active learning technique. International Journal of Nursing Education Scholarship. 2005; 2: 1-13. https: //doi.org/10.2202/1548-923X.1116

[2] Keatley VM. Identifying and articulating the characteristics of nursing agency: BSN students' perspective. Self-Care, Dependent-Care \& Nursing. 2008; 16: 18-21.

[3] Kobert LJ. In our own voice: Journaling as a teaching/learning technique for nurses. Journal of Nursing Education. 1995; 34(3): 140142.

[4] Lasater K, Nielsen A. Reflective journaling for clinical judgment development and evaluation. Journal of Nursing Education. 2009; 48 : 40-44. https : //doi .org/10.3928/01484834-20090101-06

[5] Lauterbach SS, Hentz PB. Journaling to learn: a strategy in nursing education for developing the nurse as person and person as nurse. International Journal for Human Caring. 2005; 9: 29-35. https://doi.org/10.20467/1091-5710.9.1.30

[6] Dunlap JC. Using guided reflective journaling activities to capture students' changing perceptions. TechTrends. 2006; 50: 20-26. https://doi .org/10.1007/s11528-006-7614-x

[7] O'Reilly SL, Milner J. Transitions in reflective practice: Exploring student development and preferred methods of engagement. Nutrition \& Dietetics. 2015; 72(2): 150-155. https ://doi .org/10.1111/ 1747-0080. 12134

[8] Schön DA. Educating the reflective practitioner: toward a new design for teaching and learning in the professions. San Francisco, CA: Jossey-Bass; 1987; 355.

[9] Fakude LP, Bruce JC. Journaling: A quasi-experimental study of student nurses' reflective learning ability. Curationis. 2003; 26: 49-55. https://doi.org/10.4102/curationis.v26i2.783

[10] Ruiz-Lopez M, Rodriguez-Garcia M, Villanueva PG, et al. The use of reflective journaling as a learning strategy during the clinical rotations of students from the faculty of health sciences: an action-research study. Nurse Education Today. 2015; 35: 26-31. PMid:26276531 https://doi.org/10.1016/j.nedt.2015.07.029

[11] Tanner CA. Thinking like a nurse: a research-based model of clinical judgment in nursing. Journal of Nursing Education. 2006; 45(6): 204-211.

[12] Pan S. Analysis of newspapers travelers' tales to help students learn tourism motivations. Journal of Teaching in Travel \& Tourism. 2009; 9(3-4): 216-229. https ://doi.org/10.1080/15313220903379 281

[13] Mancall P. Bringing the world to early modern Europe: travel accounts and their audiences. Leiden, Netherlands: Kommklijke Brill NV; 2006; 168.
[14] Elsner J, Rubies JP. Voyages and visions: towards a cultural history of travel. London, UK: Reaktion Books; 1999; 344.

[15] Heuer L, Russell L, Kahlstorf H. Short-term cultural immersion in Russia. The Prairie Rose. 1997; 66(1): 7-11.

[16] Leininger MM, McFarland MR. Culture care diversity and universality: a worldwide nursing theory. 2nd ed. Sudbury, MA: Jones and Bartlett; 2006; 413.

[17] McFarland MR, Wehbe-Alamah HB. Leininger's culture care, diversity and universality: a worldwide nursing theory (3rd ed.). Burlington, MA: Jones \& Bartlett Learning; 2015; 612.

[18] Schuessler JB, Wilder B, Byrd LW. Reflective journaling and development of cultural humility in students. Nursing Education Perspectives 2012; 33(2): 96-99. https ://doi.org/10.5480/1536-5026-3 3.2.96

[19] Epp S. The value of reflective journaling in undergraduate nursing education: A literature review. International Journal of Nursing Studies. 2008; 45(9): 1379-1388. https://doi.org/10.1016/j.ij nurstu. 2008.01.006

[20] Williams GB, Gerardi MB, Gill S L, et al. Reflective journaling: innovative strategy for self-awareness for graduate nursing students. International Journal for Human Caring. 2009; 13: 36-43. https://doi.org/10.20467/1091-5710.13.3.36

[21] Sewell EA. Journaling as a mechanism to facilitate graduate nurses' role transition. Journal for Nurses in Staff Development. 2008; 24: 49-52. PMid:18391659 https://doi.org/10.1097/01.NND.0 000300862.75244 .bf

[22] Hayman B, Wilkes L, Jackson D. Journaling: Identification of challenges and reflection on strategies. Nurse Researcher. 2012; 19: $27-$ 31. https://doi.org/10.7748/nr2012.04.19.3.27.c9056

[23] Van Horn R, Freed S. Journaling and dialogue pairs to promote reflection in clinical nursing education. Nursing Education Perspectives. 2008; 29(4): 220-225.

[24] Hendrix TJ, O’Malley M, Sullivan C, et al. Nursing student perceptions of reflective journaling: a conjoint value analysis. International Scholarly Research Network. 2012; 1-8. https : //doi.org/10.5 402/2012/317372

[25] Mezirow J. Transformative learning as discourse. Journal of Transformative Education. 2003; 1: 58-63. https ://doi .org/10.117 $7 / 1541344603252172$

[26] Kitchenham A. The evolution of John Mezirow's transformative learning theory. Journal of Transformative Education. 2008; 6(2): 104-123. https://doi.org/10.1177/1541344608322678

[27] De Chesnay M. Nursing research using data analysis: Qualitative designs and methods in nursing. Springer Publishing Co. 2015. Available from: https://ebookcentral.proquest.com https : //doi.org/10.1891/9780826126894 